\title{
Generalized Fixed Drug Eruption Induced by Fluconazole Without Cross-Reactivity to Itraconazole: Lymphocyte Transformation Test Confirms the Diagnosis
}

\author{
Semra Demir ${ }^{1} \cdot$ Esin Aktas Cetin $^{2} \cdot$ Derya Unal $^{1} \cdot$ Raif Coşkun $^{1} \cdot$ Muge Olgac $^{1} \cdot$ \\ Asli Gelincik $^{1}$ - Bahauddin Colakoglu ${ }^{1} \cdot$ Suna Buyukozturk ${ }^{1}$
}

Published online: 2 January 2018

(c) The Author(s) 2018. This article is an open access publication

\begin{abstract}
We present a rare case of generalized fixed drug eruption caused by fluconazole. A 45-year-old female patient was referred to our outpatient clinic because of suspicious drug eruptions that occurred 5 months earlier and resolved within a month. The patient had sequela of hyperpigmentation on her arms, legs, back, and abdomen after oral administration of the fourth dose of $150 \mathrm{mg}$ of fluconazole once daily because of vaginal candidiasis. Patch tests with the culprit drug applied both on unaffected skin areas and over one of the lesions were negative. A lymphocyte transformation test was performed and in response to fluconazole, $\mathrm{CD} 4^{+} \mathrm{T}$ cells significantly proliferated. Because the patient needed a safe antifungal drug for her recurrent vaginal candidiasis symptoms, a singleblind placebo-controlled drug provocation test was performed with itraconazole and was negative. Accordingly, $200 \mathrm{mg}$ of itraconazole once daily was given for 10 days safely.
\end{abstract}

Semra Demir

ertansemra@yahoo.com

1 Division of Immunology and Allergic Diseases, Department of Internal Medicine, Istanbul Faculty of Medicine, Istanbul University, Millet Street, Capa, Istanbul, Turkey

2 Department of Immunology, Aziz Sancar Institute of Experimental Medicine, Istanbul University, Istanbul, Turkey

\section{Key Points}

A lymphocyte transformation test confirms the hypersensitivity reaction induced by fluconazole.

Itraconazole can be used as an alternative in fluconazole-hypersensitive patients after a drug provocation test.

\section{Introduction}

A fixed drug eruption (FDE) is an adverse drug reaction characterized by skin lesions recurring on the same sites upon re-exposure to a drug. It is primarily observed within a few hours to days of drug exposure and typically resolves with hyperpigmentation [1]. Although a single lesion is usually seen, multiple eruptions can also develop [2]. The most common causes of FDEs are antibacterial drugs, nonsteroidal anti-inflammatory drugs, phenolphthalein, and related compounds [2].

Fluconazole is a triazole antifungal drug frequently used for the treatment of oral, esophageal, or vaginal candidiasis and the common adverse reactions include diarrhea, nausea, abdominal pain, headache, and skin rash. The uncommon adverse reactions are agranulocytosis, hair loss, paresthesia, anaphylaxis, vertigo, and FDEs [3]. We present a rare case of a generalized FDE caused by fluconazole in whom diagnosis was confirmed by a lymphocyte transformation test (LTT) and itraconazole was used safely.. 


\section{Case Report}

A 45-year-old female patient was referred to our outpatient clinic because of suspicious drug eruptions that occurred 5 months earlier and resolved within a month with sequela of hyperpigmentation after fluconazole administration because of vaginal candidiasis. After the fourth dose of $150 \mathrm{mg}$ (once a day orally) of fluconazole, erythematous itchy oval multiple lesions on the arms, legs, back, and abdomen developed. The patient did not use any drug simultaneously. She stopped using the drug and applied a topical corticosteroid on the lesions and they resolved with hyperpigmentation (Fig. 1). She did not take fluconazole again.

The biopsy taken from the hyperpigmented area revealed only increased melanocytes. Patch tests with fluconazole diluted in petrolatum to obtain the drug concentration of $30 \%$ were applied on the upper back and over the lesions. The tests, which were read 20 min after application, as well as over the next 3 consecutive days and 1 week later, were negative [4].

Because the patient did not want to be provocated with the culprit drug to clarify the reaction, a LTT was performed. Peripheral blood mononuclear cells were isolated by Ficoll-Hypaque (Sigma Chemical Company, St. Louis,
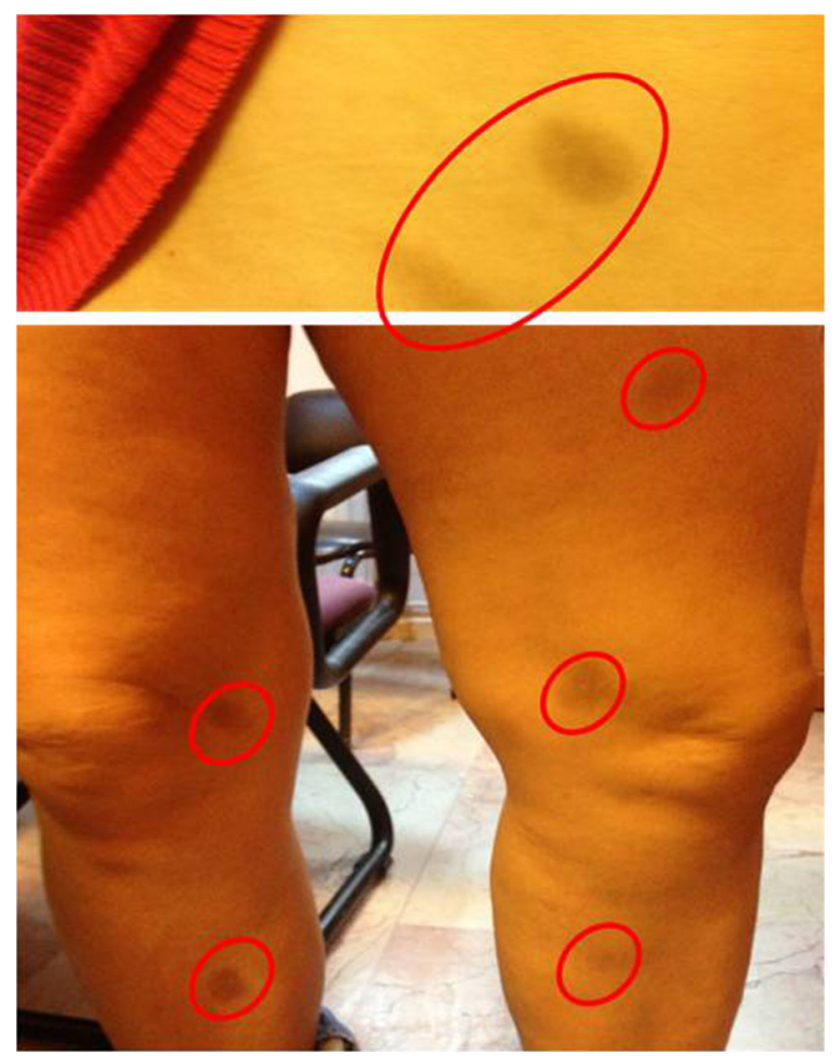

Fig. 1 Hyperpigmented skin lesions developed as a result of fluconazole
MO, USA) density gradient centrifugation. Cells were cultured with 2.5-, 5-, 10-, 20-, and $40-\mu \mathrm{g} / \mathrm{mL}$ doses of fluconazole (Lumen, Mustafa Nevzat, Turkey) and unstimulated conditions for 5 days. Then, cells were analyzed using FACSCalibur (BD Biosciences, San Jose, CA, USA) running BD CellQuest software (eBioscience, San Diego, CA, USA) and FlowJo software (TreeStar, San Carlos, CA, USA). Fluconazole-specific $\mathrm{CD}^{+}{ }^{+}$T-cell proliferation greater than twice the proliferation of unstimulated cells was accepted as positive. T-cell proliferation increased by the addition of $40 \mu \mathrm{g} / \mathrm{mL}$ of fluconazole to the peripheral lymphocyte culture with increases of 2.55 (Fig. 2). As the patient needed a safe antifungal drug owing to her recurrent vaginal candidiasis, a single-blind placebo-controlled drug provocation test starting with a one-eighth dilution of $200 \mathrm{mg}(25 \mathrm{mg}$ ) of itraconazole was given in four doses of $50 \mathrm{mg}$ every other $6 \mathrm{~h}$ and was found negative. Itraconazole was then given for 10 days (200 mg once a day orally) because of recurrent vaginal candidiasis. Itraconazole in its oral form could not be homogenized and was found unsuitable for a LTT.

\section{Discussion}

In this case report, we described a case of a generalized FDE caused by fluconazole in whom the diagnosis was confirmed by a LTT for the first time. According to the current literature, fluconazole can infrequently induce a FDE. Fluconazole was not reported as a causative agent for a FDE in a retrospective epidemiological study from Singapore conducted between 2008 and 2012 [1]. In another study performed in Bangladesh, fluconazole was responsible for $4.16 \%$ of the reactions and was the seventh most common cause of the FDE, which showed that more cases appeared to be responsible than previously recognized [5].

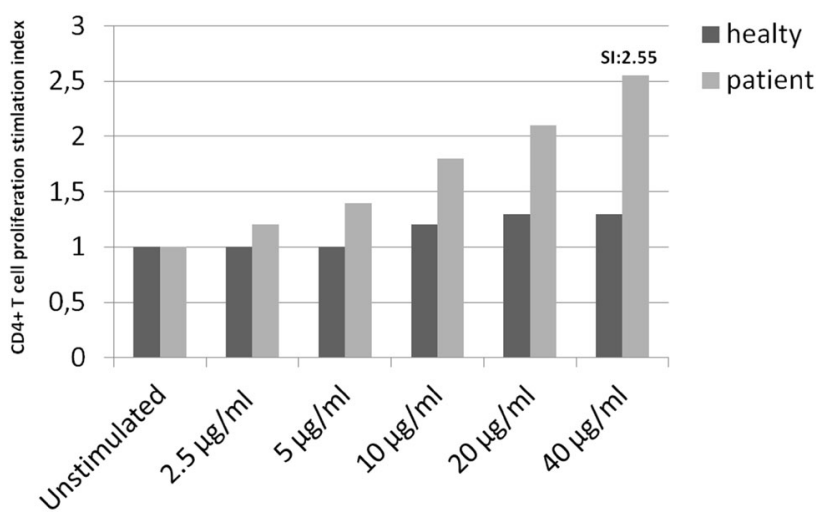

Fig. 2 Lymphocyte transformation test results of fluconazole-specific $\mathrm{CD} 4^{+} \mathrm{T}$ cells. The bar graphs represent the stimulation index (SI) of proliferating $\mathrm{CD}^{+} \mathrm{T}$ cells under different drug concentrations 
In the present case, neither histopathology nor the skin testing revealed a clue to confirm the diagnosis. Because the patient needed an antifungal drug for recurrent vaginal candidiasis, we decided to provocate her with the culprit drug but she did not give consent. In-vitro tests that could confirm the diagnosis of a FDE due to fluconazole were not previously reported. We performed a LTT in increasing doses as suggested [6]. At the dose of $40 \mu \mathrm{g} / \mathrm{mL}$ of fluconazole, significant CD4reactions are agranulocytosis T-cell proliferation was observed (Fig. 2).

A study evaluating the utility of patch testing with different drugs in drug eruptions reported a positivity rate of $20 \%$ in FDEs [7]. Moreover, there are no reports of a LTT with fluconazole. Mayorga et al. reviewed articles studying LTT with different drugs and reported the sensitivity and specificity as 65.1 and $96.5 \%$, respectively, in mild and moderate reactions [8]. As these studies indicate that a LTT has a better diagnostic value, we speculate that our results are in concordance with the literature.

Although a drug provocation test is the gold standard method to diagnose drug hypersensitivity reactions, reexposure can lead to the re-occurrence of symptoms. Therefore, an in-vitro test can be helpful to confirm the diagnosis as in this case. In a drug allergy work-up, one of the main issues is to detect or exclude cross-reactivity between similar chemical molecules. Because the hypersensitivity reactions due to triazole drugs are not so common, the rate of cross-reactivity between these drugs can be conflicting among different studies $[9,10]$. Triazoles are composed of two carbon atoms, three nitrogen atoms as well as different side chains, and the molecular formulae of fluconazole and itraconazole are $\mathrm{C}_{13} \mathrm{H}_{12} \mathrm{~F}_{2} \mathrm{~N}_{6} \mathrm{O}$ and $\mathrm{C}_{35}$ $\mathrm{H}_{38} \mathrm{Cl}_{2} \mathrm{~N}_{8} \mathrm{O}_{4}$, respectively [11, 12]. These differences in structure may explain the variety in the cross-reactivity results among different triazole drugs.

\section{Conclusion}

This case report suggests that a LTT can be used to confirm fluconazole as the causative agent for delayed-type reactions including a generalized FDE, and itraconazole can be used alternatively to treat fungal infections after a drug provocation test. Studies using both in-vivo and in-vitro diagnostic methods among larger patient numbers will further elucidate the importance of these tests in the diagnosis of hypersensitivity reactions due to triazole drugs.

Funding No financial support was received for the preparation of this article.

\section{Compliance with Ethical Standards}

Conflict of interest Semra Demir, Esin Aktas Cetin, Derya Unal, Raif Coşkun, Muge Olgac, Asli Gelincik, Bahauddin Colakoglu, and Suna Buyukozturk have no conflicts of interest directly relevant to the content of this article.

Consent to participate Written informed consent was obtained from the patient for the publication of this case report and the accompanying images.

Open Access This article is distributed under the terms of the Creative Commons Attribution-NonCommercial 4.0 International License (http://creativecommons.org/licenses/by-nc/4.0/), which permits any noncommercial use, distribution, and reproduction in any medium, provided you give appropriate credit to the original author(s) and the source, provide a link to the Creative Commons license, and indicate if changes were made.

\section{References}

1. Heng YK, Yew YW, Lim DSY, Lim YL. An update of fixed drug eruptions in Singapore. J Eur Acad Dermatol Venereol. 2015;29(8):1539-44.

2. Breathnach SM. Drug reactions. In: Burns T, Breathnach S, Cox N, Griffiths C, editors. Rook's textbook of dermatology. Oxford: Blackwell Publishing; 2010. pp. 28-30.

3. Adverse reactions. Fluconazole: drug information. UpToDate ${ }^{\circledR}$. $\mathrm{http}: / / \mathrm{www}$.uptodate.com/contents/fluconazole-druginformation? source $=$ search_result\&search=fluconazole $\% 20$ drug $\% 20$ information \&selectedTitle=1 150\#F171644. Accessed 23 Dec 2017.

4. Barbaud A, Gançalo M, Bruynzeel D, Bircher A. Guidelines for performing skin tests with drugs in the investigation of cutaneous adverse drug reactions. Contact Dermatitis. 2001;45(6):321-8.

5. Rahman MH. Fixed drug eruption in Bangladeshi population: confirmed by provocative test. Int J Dermatol. 2014;53(2):255-8. https://doi.org/10.1111/ijd.12197.

6. Pichler WJ, Tilch J. The lymphocyte transformation test in the diagnosis of drug hypersensitivity. Allergy. 2004;59(8):809-20.

7. Ohtoshi S, Kitami Y, Sueki H, Nakada T. Utility of patch testing for patients with drug eruption. Clin Exp Dermatol. 2014;39(3):279-83. https://doi.org/10.1111/ced.12239.

8. Mayorga C, Doña I, Perez-Inestrosa E, Fernández TD, Torres MJ. The value of in vitro tests to diminish drug challenges. Int $\mathrm{J}$ Mol Sci. 2017;18(6):E1222. https://doi.org/10.3390/ijms18061222.

9. Bavbek S, Yılmaz I, Sözener ZÇ. Fixed drug eruption caused by ornidazole and fluconazole but not isoconazole, itraconazole, ketoconazole and metronidazole. J Dermatol. 2013;40(2):134-5.

10. Benjamin Lash D, Jolliff J, Munoz A, Heidari A. Cross-reactivity between voriconazole, fluconazole and itraconazole. J Clin Pharm Ther. 2016;41:566-7.

11. National Center for Biotechnology Information. PubChem compound database. http://pubchem.ncbi.nlm.nih.gov/compound/ 3365. Accessed 23 Dec 2017.

12. National Center for Biotechnology Information. PubChem compound database. Available from: http://pubchem.ncbi.nlm.nih. gov/compound/55283. Accessed 23 Dec 2017. 